\title{
Pandemic in years
}

(BIRDEM Med J 2020; 10(2): 84-86)

\section{COVID-19}

Since $31^{\text {st }}$ December, 2020 people largely could learn the name corona. The new (novel corona virus 2019) virus is a version of the zoonotic virus. Subsequently, it has been named COVID-19 (corona virus infected disease). It is actually SARS-CoV 2, named after its predecessor SARS-CoV, the virus responsible for 2002 outbreak of SARS (severe acute respiratory distress), a similar one is Middle East acute respiratory distress syndrome (MARS) of Middle East with an outbreak in 2012. It appears that, COVID-19 spreads from a wet market of raw fish, meat and vegetable in Wuhan city of Hubei province of China. The virus corona is responsible for $10-30 \%$ of seasonal flue like illness throughout the globe. Like other viruses, it gets mutated, changes characteristic and causes human catastrophe, the pandemic.

Mild disease (influenza like illness, ILI): Through social media, medical journals, affected persons, all over the world know that $80 \%$ suffer from mild disease like seasonal flu-like symptoms, includes cough, coryza, rhinorrhea, sneezing and fever. When there is continuous uninterrupted and distressing cough in this period with fever or rising body temperature, someone should think of COVID-19. Patient feel very week and exhausted. Sore throat is another feature to be taken into account, feeling pain in talking, swallowing and coughing.

Moderate (pneumonia): When patients develop pneumonia, there is chest pain, difficulty in breathing becomes pronouncing and higher degree of fever.

Severe disease (SARI) - severe pneumonia, ARDS, septicemia, septic shock): Severity increases and patients get worse from severe pneumonia to ARDS (acute respiratory distress syndrome) to septicemia and shock.

\section{Case definitions}

WHO periodically updates the Global Surveillance for human infection with COVID-19 document, which includes case definitions. For easy reference, cases has been categorized as 1 . suspected cases 2 . probable cases and 3. Confirmed case. It is used in isolation, quarantine and treatment stratification of corona cases.

\section{Laboratory tests for COVID-19 cases}

1. methods that detect the presence of virus

2. those that detect antibodies produced in response to infection

3. tests to evaluate corona effect in a patient

\section{Methods that detect the presence of virus}

RT-PCR detects the coronavirus' RNA. This test is specific and is designed to only detect the RNA of the SARS-CoV2 virus. It is used to confirm very recent or active infections. PCR is two step - exrraction and detection. It takes hours to days. This is a process of time-consuming series of alternating temperature cycles (thermal) variation. Initially, globally there was shortage of test kits.

There appeared a competition of companies how rapid they can test and how many samples they can perform each day. Since March 2020 FDA has approved different companies to do this. Cepheid has a test method which gives result in 45 minutes, Abbott lab can make it in 5 minutes, they uses isothermal technology. FDA has approved these as EUA (emergency use authorization).

Antigen test is a test, which uses a monoclonal antibody which specifically binds to the nucleocapsid protein ( $\mathrm{N}$ protein) of the novel coronavirus, is being developed in Taiwan, with the hope that it can provide results in 15 to 20 minutes just like a rapid influenza test (corona virus has 4 proteins in cover, one has been used).

All these tests are technician dependent and the collection of sample is difficult. It should be taken from posterior wall of nasopharynx within 7 days of infection for better result.

Antibody test, as of $15^{\text {th }}$ April, 2020, four tests had been approved for diagnosis in the United States, all under FDA EUA. In late March 2020, a number of companies received European approval for their test kits. 
The testing capacity is several hundred samples within hours and therefore, much faster than the conventional PCR assay of viral RNA. The most, if not all, of the current COVID-19 antibody testing done at large scale is for detection of binding antibodies only and does not measure neutralizing antibodies, so, these tests can not assure protection.

Antigen antibody test, In Bangladesh, Gonoshyastho has developed a system to test presence of antigen and antibody with single kit. This is a blood test. This point of care will give result in half an hour. Currently, it is awaiting for approval by the Government.

\section{Chest CT scans and radiographs}

A March 2020 literature review concluded that chest radiographs are of little diagnostic value in early stages and CT chest may be performed even before symptom onset.

Typical features on CT include bilateral multilobar ground-glass opacificities with a peripheral, asymmetric and posterior distribution with subpleural dominance, crazy paving and consolidation develop as the disease evolves. A study comparing PCR to CT in Wuhan at the point of origin of the current pandemic has suggested that $\mathrm{CT}$ is significantly more sensitive than PCR, though less specific.

\section{Human readers and artificial intelligence}

Artificial intelligence based convolutional neural networks have also been developed to detect imaging features of the virus both on radiographs and CT with significantly higher specificity. As of March 2020, the CDC recommends PCR for initial screening because it has higher specificity than CT chest.

\section{Supporting investigations}

CBC shows normal or low total white cell counts with lymphopenia. If ratio of lymphocyte less than 4 relative to neutrophil, it indicates poor prognosis. High ferritin, CRP and d-Dimer is observed, high d-Dimer indicates a severe case. Organ failure should be tested accordingly, as also, comorbidity in all hospitalized cases.

\section{Treatment}

Influenza like illness should be treated symptomatically remaining at home.

\section{Hospitalization}

Shortness of breath is an indication of hospitalization presuming a case of pneumonia. Cases can be evaluated by breathing capacity, tachypnea, lung sign pulse oxymetry, $\mathrm{x}$-ray chest. Clinically lungs may be normal but $\mathrm{x}$-ray will show shadow; oxygen saturation should be more than $95 \%$ unaided. Broad spectrum antibiotic to be given as superadded infection is frequent.

Antivirals: There is no remedy for virus as yet.

Oxygen should be given high flow to maintain PO2 95\% $(92 \%)$. Idea should be to try up to last avoiding ventilation if ARDS, respiratory failure.

Non ventilator ICU supports: High flow oxygen, proning, NIV (BiPAP), mechanical ventilator.

Anticoagulant should be given from beginning to all hospitlised patients.

Anti-inflammatory drugs: corticosteroids (dexamethasone, methyl prednisolone), toclizumab may be given in ARDS cases, as there is cytokine storm. Corticosteroid should be given prior to tocilizumab.

Fluid: adequate fluid should be given for taking care of AKI, ARDS with crucial monitoring.

\section{Prevention}

Social distancing, hand washing, quarantine, isolation and lockdown are the methods with proven effect to prevent the disease. Effective vaccine could not be developed so far, though there was disastrous outbreaks of pandemics in decades.

\section{Pandemics in decades}

A. Influenza flus (a) Spanish flu (also known as 1918 Asian flu) caused by H1N1 from January 1918 to December 1920. 500 million people affected with fatality of 50 million.

(b) Asian influenza (also known as 1957 flu pandemic) caused by H2N2 from 1957 February to June 1958, affected one million to two million with casualty around 70,000.

(c) Hong Kong flu caused by H3N3, from 13 July 1968 to December 1968 and returned the following years, in late 1969 and early 1970 and in 1972 . The Hong Kong flu yielded a low death rate, $15 \%$ of the Hong Kong residents.

(d) Pandemic 09 H1N1 (Swine flu), caused by H1N1from Jan 2009 to August 2010, peaked in April-June. It affected 700 million - 1.4 billion with death of 18,036, originated in North America. 


\section{B. Corona flu}

(a) SARS-corona caused by SARS CoV-1, Nov 2002 to July 2003, affected 8000 people with $10 \%$ casualty, originated in Guangdong, China.

(b) MERS, caused by MERS corona from 2012 to till date, mostly in Middle East camels. It affected 2519 people, so far died $866(30 \%)$.

(c) COVID-19, caused by SARS CoV-2 from Dec 2019 till date, originated in Wuhan, China. Devastated whole world. As of today affected 2.8 million with death 200000. (highest in USA)

\section{Pandemic in centuries}

a) Plague in 1720, great plague of Marseille, France killed total 100,000 people, still reminds us the embankments of quarantine with corona outbreak could bring again in front specially in Europe. b) Cholera in $\mathbf{1 8 2 0}$ or Asiatic cholera took 30000 life in Bangkok and affected around 2 million in British India.

c) Spanish flu in 1920 took 50 million lives.

d) Corona flu in $\mathbf{2 0 2 0}$ as today (30.4.2020) took 2.3 lakh life with 2.5 million affected all over the world.

Advanced developments in sciences is not yet sufficient to protect the mankind from natural infections. The plague and the cholera have all been conquered by invention of remedies. Hopefully, days will be coming to overcome the flus.

\section{References}

1. https://www.who.int/emergencies/diseases/novelcoronavirus-2019/situation-reports/ However, once local or community transmission

2. https://www.who.int/emergencies/diseases/novelcoronavirus-2019/situation-reports/ 